\title{
A NEW CONSTRUCTION FOR REGULAR SEMIGROUPS WITH QUASI-IDEAL ORTHODOX TRANSVERSALS
}

\author{
XIANGJUN KONG ${ }^{\bowtie}$ and XIANZHONG ZHAO \\ (Received 25 November 2006; accepted 12 December 2007)
}

Communicated by M. G. Jackson

\begin{abstract}
In any regular semigroup with an orthodox transversal, we define two sets $R$ and $L$ using Green's relations and give necessary and sufficient conditions for them to be subsemigroups. By using $R$ and $L$, some equivalent conditions for an orthodox transversal to be a quasi-ideal are obtained. Finally, we give a structure theorem for regular semigroups with quasi-ideal orthodox transversals by two orthodox semigroups $R$ and $L$.
\end{abstract}

2000 Mathematics subject classification: primary 20M10.

Keywords and phrases: regular semigroup, inverse transversal, orthodox transversal, quasi-ideal.

\section{Introduction and preliminaries}

The concept of inverse transversal of a regular semigroup was first introduced by Blyth and McFadden in 1982 [3]. Since then, this class of regular semigroups has attracted several authors' attention and a series of important results have been obtained [1-3, 8-11]. If $S$ is a regular semigroup, then an inverse transversal of $S$ is an inverse subsemigroup $S^{o}$ such that $S^{o}$ meets $V(a)$ precisely once for each $a \in S$ (that is, $\left|V(a) \cap S^{o}\right|=1$ ), where $V(a)=\{x \in S \mid a x a=a$ and $x a x=x\}$ denotes the set of inverses of $a$. The intersection of $V(a)$ and $S^{o}$ is denoted by $V_{S^{o}}(a)$ and the unique element of $V_{S^{o}}(a)$ is denoted by $a^{o}$. It is well known that the sets $I=\left\{e \in S \mid e e^{o}=e\right\}$ and $\Lambda=\left\{f \in S \mid f^{o} f=f\right\}$ are left regular and right regular bands, respectively, and play an important role in the study of regular semigroups with inverse transversals. Other interesting subsets of $S$ are $R=\left\{x \in S \mid x^{o} x=x^{o} x^{o o}\right\}$ and $L=\left\{x \in S \mid x x^{o}=x^{o o} x^{o}\right\}$. Both $R$ and $L$ are subsemigroups with $R$ left inverse (or $\mathcal{R}$-unipotent) and $L$ right inverse (or $\mathcal{L}$-unipotent). Moreover, $R \cap L=S^{o}$ and $E(R)=I, E(L)=\Lambda$, where $E(S)$ denotes the idempotents of $S$. By using $R$ and $L$, Saito $[9,10]$ gave some structure theorems of regular semigroups with inverse

(c) 2009 Australian Mathematical Society 1446-7887/2009 \$16.00 
transversals, while Blyth and Almeida Santos [1,2] classified the inverse transversals and gave some equivalent conditions for the inverse transversal $S^{o}$ to be a quasiideal (defined below). Orthodox transversals were introduced by Chen [4] as a generalization of inverse transversals, and an excellent structure theorem for regular semigroups with quasi-ideal orthodox transversals was also given. Afterwards, Chen and Guo [5] considered the general case of orthodox transversals and investigated some properties concerning the sets $I$ and $\Lambda$. Similarly two sets $R$ and $L$ (defined below) are shown to play an important role in the study of orthodox transversals. In this paper, we investigate some properties concerning $R$ and $L$, and obtain some results that are parallel to the corresponding results on regular semigroups with inverse transversals. The main objective of this paper is to give a structure theorem for the class of regular semigroups with quasi-ideal orthodox transversals.

In a previous publication [7] we constructed regular semigroups with quasi-ideal orthodox transversals by a formal set $(B, R)$, where $R$ is a regular semigroup with a right ideal orthodox transversal $S^{o}$ and $B$ a band with a left ideal orthodox (in fact, band) transversal $E^{o}$. Evidently, there are different conditions on the structural 'brick' $B$ and $R$. The present paper corrects this asymmetry by giving a new construction of regular semigroups with quasi-ideal orthodox transversals by way of two regular semigroups $R$ and $L$. The semigroups $R$ and $L$ share a common orthodox transversal $S^{o}$, which is a right ideal of $R$ and a left ideal of $L$. Many of the conditions on $R$ and $L$ are symmetric and one is weaker than that in [7] (that is, if $x \in S^{o}$ or $a \in S^{o}$ then $a * x=a x$ in this paper; instead of if $x \in E^{o}$ or $e \in E^{o}$, then $e * x=e x$ in [7]).

Let $S$ be a semigroup and $S^{o}$ a subsemigroup of $S$. Then $S^{o}$ is said to be an orthodox transversal of $S$ if the following conditions are satisfied.

(1.1) For all $a \in S, V_{S^{o}}(a) \neq \emptyset$.

(1.2) If $a, b \in S$ and $\{a, b\} \cap S^{o} \neq \emptyset$, then $V_{S^{o}}(a) V_{S^{o}}(b) \subseteq V_{S^{o}}(b a)$.

Note that, if $S^{o}$ is an orthodox transversal of $S$, then $S$ is a regular semigroup by (1.1) and $S^{o}$ is an orthodox subsemigroup of $S$ by (1.2).

A subsemigroup $S^{o}$ of $S$ is said to be a quasi-ideal of $S$ if $S^{o} S S^{o} \subseteq S^{o}$.

The following theorem will be frequently used without further mention.

(1.3) Let $e$ and $f$ be $\mathcal{D}$-equivalent idempotents of a semigroup $S$. Then each element $a$ of $R_{e} \cap L_{f}$ has a unique inverse $a^{\prime}$ in $R_{f} \cap L_{e}$, such that $a a^{\prime}=e$ and $a^{\prime} a=f$.

(1.4) Let $a, b$ be elements of a semigroup $S$. Then $a b \in R_{a} \cap L_{b}$ if and only if $L_{a} \cap R_{b}$ contains an idempotent.

Finally, we list two basic results that are used in this paper.

LEMMA 1.1. [5] Let $S^{o}$ be a subsemigroup of $S$ and $V_{S^{o}}(a) \neq \emptyset$ for each $a \in S$. Then the following conditions are equivalent:

(1) $S^{o}$ is an orthodox transversal of $S$;

(2) $I E\left(S^{o}\right) \subseteq I, E\left(S^{o}\right) \Lambda \subseteq \Lambda, E\left(S^{o}\right) I \subseteq E(S), \Lambda E\left(S^{o}\right) \subseteq E(S)$. 
LEMMA 1.2. [5] Let $S^{o}$ be an orthodox transversal of $S$. Then the following conditions are equivalent:

(1) I is a band;

(2) $E\left(S^{o}\right) I \subseteq I$;

(3) $(\forall f \in I)\left(\exists f^{*} \in E\left(S^{o}\right), f^{*} \mathcal{L} f\right) f^{*} E\left(S^{o}\right) f \subseteq E\left(S^{o}\right)$;

(4) $(\forall f \in I)\left(\forall f^{*} \in E\left(S^{o}\right), f^{*} \mathcal{L} f\right) f^{*} E\left(S^{o}\right) f \subseteq E\left(S^{o}\right)$.

We adopt the terminology and notation of $[4,6,8]$.

\section{Some properties}

We begin this section by investigating some elementary properties of the sets $R$ and $L$. For any result concerning $R$ there is a dual result for $L$, which we list but omit its proof.

THEOREM 2.1. Let $S$ be a regular semigroup with an orthodox transversal $S^{o}$. Let

$$
\begin{aligned}
R & =\left\{x \in S \mid\left(\forall x^{o} \in V_{S^{o}}(x)\right)\left(\exists x^{o o} \in V_{S^{o}}\left(x^{o}\right)\right) x^{o} x=x^{o} x^{o o}\right\}, \\
L & =\left\{a \in S \mid\left(\forall a^{o} \in V_{S^{o}}(a)\right)\left(\exists a^{o o} \in V_{S^{o}}\left(a^{o}\right)\right) a a^{o}=a^{o o} a^{o}\right\} .
\end{aligned}
$$

Then

$$
\begin{aligned}
R & =\left\{x \in S \mid\left(\exists y^{o} \in V_{S^{o}}(x), \exists y^{o o} \in V_{S^{o}}\left(y^{o}\right)\right) y^{o} x=y^{o} y^{o o}\right\} \\
& =\left\{x \in S \mid\left(\exists e^{o} \in E^{o}\right) x \mathcal{L} e^{o}\right\}, \\
L & =\left\{a \in S \mid\left(\exists b^{o} \in V_{S^{o}}(a), \exists b^{o o} \in V_{S^{o}}\left(b^{o}\right)\right) a b^{o}=b^{o o} b^{o}\right\} \\
& =\left\{a \in S \mid\left(\exists f^{o} \in E^{o}\right) a \mathcal{R} f^{o}\right\} .
\end{aligned}
$$

PROOF. It is evident that

$$
R=\left\{x \in S \mid\left(\forall x^{o} \in V_{S^{o}}(x)\right)\left(\exists x^{o o} \in V_{S^{o}}\left(x^{o}\right)\right) x=x x^{o} x^{o o}\right\} .
$$

For the first equation, we only need to show that, for $x \in S$, if there exist $y^{o} \in V_{S^{o}}(x), y^{o o} \in V_{S^{o}}\left(y^{o}\right)$ such that $y^{o} x=y^{o} y^{o o}$, then $x \in R$. We notice that $x \mathcal{L} y^{o} x=y^{o} y^{o o}$ since $y^{o} \in V_{S^{o}}(x)$. For $x^{o}, y^{o} \in S^{o}, x \in V\left(x^{o}\right) \cap V\left(y^{o}\right) \neq \emptyset$, by [5, Lemma 2.2] we have $V_{S^{o}}\left(x^{o}\right)=V_{S^{o}}\left(y^{o}\right)$, so $y^{o o} \in V_{S^{o}}\left(x^{o}\right)$. So $x \mathcal{L} y^{o} y^{o o} \mathcal{L} x^{o} y^{o o}$ and hence $x=x x^{o} y^{o o}$. That is, $x \in R$.

For the second equation, if $x \in R$, then $x \mathcal{L} x^{o} x=x^{o} x^{o o} \in E\left(S^{o}\right)$. Conversely, if there exists $e^{o} \in E^{o}$ such that $x \mathcal{L} e^{o}$, then for any $x^{o} \in V_{S^{o}}(x), x^{o} \mathcal{R} x^{o} x \mathcal{L} x \mathcal{L} e^{o}$, thus $x^{o} x \in E^{o}$ by [5, Theorem 2.4]. So $x^{o} x \mathcal{R}^{S^{o}} x^{o}$ and thus there exists $x^{o o} \in V_{S^{o}}\left(x^{o}\right)$ such that $x^{o} x=x^{o} x^{o o}$ since every idempotent in $R_{x^{o}}$ is of the form $x^{o} x^{o \prime}$ for some $x^{o \prime} \in V_{S^{o}}\left(x^{o}\right)$. Therefore $x \in R$, and the theorem is proved.

Notice that

$$
I=\left\{e \in E(S) \mid\left(\exists e^{*} \in E^{o}\right) e \mathcal{L} e^{*}\right\}, \quad \Lambda=\left\{f \in E(S) \mid\left(\exists f^{+} \in E^{o}\right) f \mathcal{R} f^{+}\right\},
$$

and by Theorem 2.1, we have the following result. 
Corollary. Let $R$ and $L$ be as in Theorem 2.1. Then $R \cap L=S^{o}$ and $E(R)=I$, $E(L)=\Lambda$.

As we know, $I$ and $\Lambda$ are subbands of $S$ if $S^{o}$ is an inverse transversal of $S$ (see [11]). But in general, the corresponding result fails to be true if $S^{o}$ is an orthodox transversal of $S$ (see [5]). In [5], Chen and Guo proved, in general, that if $S^{o}$ is an orthodox transversal of $S$, then the semibands $\bar{I}$ and $\bar{\Lambda}$ generated by $I$ and $\Lambda$ respectively are bands, and they also gave some equivalent conditions for $I, \Lambda$ to be bands. By $R$ and $L$, we obtain an equivalent condition for $I$ and $\Lambda$ to be bands, which is parallel to the result on regular semigroups with inverse transversals.

THEOREM 2.2. Let $S$ be a regular semigroup with an orthodox transversal $S^{o}$. Then $R(L)$ is a subsemigroup of $S$ if and only if $I(\Lambda)$ is a subsemigroup of $S$.

Proof. Suppose that $R$ is a subsemigroup of $S$. Let $e, f \in I$. Then $e, f \in R$ and so $e f \in R$ since $R$ is a subsemigroup. Also we have ef $\in E(S)$ by [9, Theorem 2.6], whence ef $\in E(S) \cap R=I$.

Conversely, suppose that $I$ is a subsemigroup of $S$ and let $x, y \in R$. Then

$$
\begin{aligned}
x y & =x x^{o} x^{o o} y y^{o} y^{o o} \\
& =x \cdot x^{o} x^{o o} y y^{o} \cdot x^{o} x^{o o} y y^{o} \cdot y^{o o} \\
& =x y \cdot y^{o} x^{o} \cdot x^{o o} y .
\end{aligned}
$$

By the definition of an orthodox transversal, we have $y^{o} x^{o} \in V_{S^{o}}\left(x^{o o} y\right)$, and so

$$
\begin{aligned}
y^{o} x^{o} \cdot x^{o o} y & =y^{o} \cdot y^{o o} y^{o} x^{o} x^{o o} y y^{o} \cdot y^{o o} \\
& \in y^{o} \cdot y^{o o} y^{o} E^{o} \cdot y y^{o} \cdot y^{o o} \\
& \subseteq y^{o} \cdot E^{o} \cdot y^{o o} \quad\left(\text { since } y y^{o} \in I, y y^{o} \mathcal{L} y^{o o} y^{o} \in E^{o}\right) \\
& \subseteq E^{o} .
\end{aligned}
$$

So we have $x y=x y \cdot y^{o} x^{o} \cdot x^{o o} y \mathcal{L} y^{o} x^{o} \cdot x^{o o} y \in E^{o}$; by Theorem 2.1, $x y \in R$.

LEMMA 2.3. Let $S$ be a regular semigroup with an orthodox transversal $S^{o}$. If $x \in R$ or $y \in L$, then $V_{S^{o}}(y) V_{S^{o}}(x) \subseteq V_{S^{o}}(x y)$.

PRoof. If $x \in R$, then for any $x^{o} \in V_{S^{o}}(x)$ there exists $x^{o o} \in V_{S^{o}}\left(x^{o}\right)$ such that $x^{o} x=x^{o} x^{o o}$. For any $y^{o} \in V_{S^{o}}(y)$,

$$
x^{o} x y y^{o}=x^{o} x^{o o} y y^{o} \in E\left(S^{o}\right) \Lambda \subseteq E(S)
$$

and

$$
y y^{o} x^{o} x=y y^{o} x^{o} x^{o o} \in I E\left(S^{o}\right) \subseteq E(S) .
$$

Thus

$$
x y \cdot y^{o} x^{o} \cdot x y=x \cdot x^{o} x y y^{o} \cdot x^{o} x y y^{o} \cdot y=x \cdot x^{o} x y y^{o} \cdot y=x y
$$


and

$$
y^{o} x^{o} \cdot x y \cdot y^{o} x^{o}=y^{o} \cdot y y^{o} x^{o} x \cdot y y^{o} x^{o} x \cdot x^{o}=y^{o} \cdot y y^{o} x^{o} x \cdot x^{o}=y^{o} x^{o} .
$$

For the choice of $x^{o}$ and $y^{o}$, we have $V_{S^{o}}(y) V_{S^{o}}(x) \subseteq V_{S^{o}}(x y)$.

THEOREM 2.4. Let $S$ be a regular semigroup with an orthodox transversal $S^{o}$. Then the following statements are equivalent:

(1) $S^{o}$ is a quasi-ideal;

(2) $E\left(S^{o}\right) I \subseteq E\left(S^{o}\right), \Lambda E\left(S^{o}\right) \subseteq E\left(S^{o}\right)$;

(3) $\Lambda I \subseteq S^{o}$;

(4) $S S^{o} \subseteq R, S^{o} S \subseteq L$;

(5) $R$ is a left ideal and $L$ is a right ideal of $S$.

Proof. Obviously, (1), (2) and (3) are equivalent.

(1) implies (4). If (1) holds, then $y x^{o} \mathcal{L}\left(y x^{o}\right)^{o} y x^{o} \in S^{o} \cap E(S)=E\left(S^{o}\right)$, whence $S S^{o} \subseteq R$; and dually $S^{o} S \subseteq L$.

(4) implies (5). If (4) holds, then for any $x \in S$ and $y \in R$, we have $x y=x y y^{o} y^{o o} \in$ $S S^{o} \subseteq R$, whence $S R \subseteq R$; and dually $L S \subseteq L$.

(5) implies (3). If (5) holds, then for $l \in \Lambda$ and $i \in I$, there exist $i^{o}, l^{o} \in E\left(S^{o}\right)$, such that $i=i i^{o}, l=l^{o} l$. Thus

$$
l i=l i i^{o} \in S S^{o} \subseteq S R \subseteq R \text { and } \quad l i=l^{o} l i \in S^{o} S \subseteq L S \subseteq L,
$$

whence $l i \in R \cap L=S^{o}$ and we have (3).

TheOREM 2.5. Suppose that $a, a^{\prime} \in L$ and $a \mathcal{L} a^{\prime}, y, y^{\prime} \in R$ and $y \mathcal{R} y^{\prime}$. Then

$$
y^{o} y^{\prime} V_{S^{o}}\left(a^{\prime} y^{\prime}\right) a^{\prime} a^{o} \subseteq V_{S^{o}}(a y),
$$

where $y^{o} \in V_{S^{o}}(y) \cap V_{S^{o}}\left(y^{\prime}\right), a^{o} \in V_{S^{o}}(a) \cap V_{S^{o}}\left(a^{\prime}\right)$.

PROOF. Take $s \in V_{S^{o}}\left(a^{\prime} y^{\prime}\right)$. Then

$$
a y\left(y^{o} y^{\prime} s a^{\prime} a^{o}\right) a y=a y^{\prime} s a^{\prime} y=a a^{o} a^{\prime} y^{\prime} s a^{\prime} y^{\prime} y^{o} y=a a^{o} a^{\prime} y^{\prime} y^{o} y=a y
$$

and

$$
\left(y^{o} y^{\prime} s a^{\prime} a^{o}\right) a y\left(y^{o} y^{\prime} s a^{\prime} a^{o}\right)=y^{o} y^{\prime} s a^{\prime} y^{\prime} s a^{\prime} a^{o}=y^{o} y^{\prime} s a^{\prime} a^{o} .
$$

\section{The main theorem}

The main objective in this section is to give a structure theorem for regular semigroups with quasi-ideal orthodox transversals. In what follows $R$ denotes a regular semigroup with a right ideal orthodox transversal $S^{o}$. Then by [7, Lemma 1], $E(R)=I$ is a band, consequently $R$ is an orthodox semigroup and we will denote the minimum inverse semigroup congruence on $R$ by $\gamma$. For $a \in R$, the $\mathcal{R}$-class of $R$ 
containing $a$ will be denoted by $R_{a}$ and the $\gamma$-class containing $a$ will be denoted by $T(a)$. Then $T(a) \cap S^{o}=V_{S^{o}}(a)$ and by [5, Theorem 2.6] and since $R$ is orthodox,

$$
\begin{aligned}
V_{S^{o}}(a) \cap V_{S^{o}}(b) \neq \emptyset \quad \Longleftrightarrow \quad V_{S^{o}}(a)=V_{S^{o}}(b) \quad \Longleftrightarrow \quad \begin{array}{r}
T(a)=T(b) \\
\text { for all } a, b \in R .
\end{array}
\end{aligned}
$$

We define $K(a)=K(b)$ if $R_{a}=R_{b}$ and $T(a)=T(b)$ for $a, b \in R$ and we define a relation $\mathcal{K}$ on $R$ by $(a, b) \in \mathcal{K}$ if $K(a)=K(b)$. Then $\mathcal{K}$ is an equivalence relation on $R$.

THEOREM 3.1. Let $R$ and $L$ be regular semigroups with a common orthodox transversal $S^{o}$. Suppose that $S^{o}$ is a right ideal of $R$ and a left ideal of $L$. Let $L \times R \longrightarrow S^{o}$ described by $(a, x) \longrightarrow a * x$ be a mapping such that for any $x, y \in R$ and for any $a, b \in L$ :

(1) $(a * x) y=a * x y$ and $b(a * x)=b a * x$;

(2) if $x \in S^{o}$ or $a \in S^{o}$, then $a * x=a x$; and

(3) if $a, a^{\prime} \in L$ and $a \mathcal{L} a^{\prime}, y, y^{\prime} \in R$ and $y \mathcal{R} y^{\prime}$, then

$$
y^{o} y^{\prime} V_{S^{o}}\left(a^{\prime} * y^{\prime}\right) a^{\prime} a^{o} \subseteq V_{S^{o}}(a * y),
$$

where $y^{o} \in V_{S^{o}}(y) \cap V_{S^{o}}\left(y^{\prime}\right), a^{o} \in V_{S^{o}}(a) \cap V_{S^{o}}\left(a^{\prime}\right)$.

Define a multiplication on the set

$$
\Gamma=R / \mathcal{K}|\times| L / \mathcal{L}=\left\{\left(K_{x}, L_{a}\right) \in R / \mathcal{K} \times L / \mathcal{L} \mid V_{S^{o}}(x) \cap V_{S^{o}}(a) \neq \emptyset\right\}
$$

by

$$
\left(K_{x}, L_{a}\right)\left(K_{y}, L_{b}\right)=\left(K_{x x^{o}(a * y)}, L_{(a * y) y^{o} b}\right) .
$$

Then $\Gamma$ is a regular semigroup with a quasi-ideal orthodox transversal that is isomorphic to $S^{o}$.

Conversely, every regular semigroup with a quasi-ideal orthodox transversal can be constructed in this way.

To prove this theorem, we give a sequence of lemmas as follows.

Lemma 3.2. The multiplication in $\Gamma$ is well defined.

Proof. First it is easy to see that $\left(K_{x x^{o}(a * y)}, L_{(a * y) y^{o} b}\right) \in \Gamma$, since

$$
(a * y)^{o} x^{o o} x^{o} \in V_{S^{o}}\left(x x^{o}(a * y)\right) \cap V_{S^{o}}\left((a * y) y^{o} b\right) \neq \emptyset .
$$

Let $x^{o}, x_{1}^{o} \in V_{S^{o}}(x) \cap V_{S^{o}}(a)$, then

$$
R_{x x^{o}(a * y)}=R_{x x_{1}^{o}(a * y)} \quad \text { and } \quad T\left(x x^{o}(a * y)\right)=T\left(x x_{1}^{o}(a * y)\right),
$$

and hence the multiplication in $\Gamma$ is not dependent on the choice of $x^{o}$. There is a dual result for $y^{o}$. 
Finally we prove that the multiplication in $\Gamma$ is not dependent on the choice of $x, a, y, b$. Let

$$
\left(K_{x}, L_{a}\right)=\left(K_{x^{\prime}}, L_{a^{\prime}}\right), \quad\left(K_{y}, L_{b}\right)=\left(K_{y^{\prime}}, L_{b^{\prime}}\right) .
$$

We then have

$$
\left(K_{x}, L_{a}\right)\left(K_{y}, L_{b}\right)=\left(K_{x x^{o}(a * y)}, L_{(a * y) y^{o} b}\right)
$$

and

$$
\left(K_{x^{\prime}}, L_{a^{\prime}}\right)\left(K_{y^{\prime}}, L_{b^{\prime}}\right)=\left(K_{x^{\prime} x^{o}\left(a^{\prime} * y^{\prime}\right)}, L_{\left(a^{\prime} * y^{\prime}\right) y^{o} b^{\prime}}\right),
$$

where $x^{o} \in V_{S^{o}}(x) \cap V_{S^{o}}\left(x^{\prime}\right)$ and $y^{o} \in V_{S^{o}}(y) \cap V_{S^{o}}\left(y^{\prime}\right)$.

Next we prove that $T\left(x x^{o}(a * y)\right)=T\left(x^{\prime} x^{o}\left(a^{\prime} * y^{\prime}\right)\right)$. Take $s \in V_{S^{o}}\left(a^{\prime} * y^{\prime}\right)$, then $y^{o} y^{\prime} s a^{\prime} a^{o} \in V_{S^{o}}(a * y)$ by (3). Since $S^{o}$ is orthodox,

$$
\begin{gathered}
y^{o} y^{\prime} s x^{o o} x^{o} \in V_{S^{o}}\left(x^{\prime} x^{o}\left(a^{\prime} * y^{\prime}\right)\right), \\
y^{o} y^{\prime} s a^{\prime} a^{o} x^{o o} x^{o}=y^{o} y^{\prime} s x^{o o} x^{o} \in V_{S^{o}}\left(x x^{o}(a * y)\right),
\end{gathered}
$$

where $x^{o o} \in V_{S^{o}}\left(x^{o}\right)$. So

$$
V_{S^{o}}\left(x x^{o}(a * y)\right) \cap V_{S^{o}}\left(x^{\prime} x^{o}\left(a^{\prime} * y^{\prime}\right)\right) \neq \emptyset
$$

and hence $V_{S^{o}}\left(x x^{o}(a * y)\right)=V_{S^{o}}\left(x^{\prime} x^{o}\left(a^{\prime} * y^{\prime}\right)\right)$, that is

$$
T\left(x x^{o}(a * y)\right)=T\left(x^{\prime} x^{o}\left(a^{\prime} * y^{\prime}\right)\right)
$$

as required.

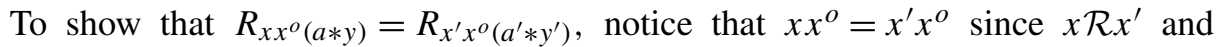
$x^{o} \in V_{S^{o}}(x)=V_{S^{o}}\left(x^{\prime}\right)$, and $x^{o} a=x^{o} a^{\prime}$ since $a \mathcal{L} a^{\prime}$ and $x^{o} \in V_{S^{o}}(a)=V_{S^{o}}\left(a^{\prime}\right)$. Take $s \in V_{S^{o}}\left(a^{\prime} * y^{\prime}\right)$, then $(a * y)^{o}=y^{o} y^{\prime} s a^{\prime} a^{o} \in V_{S^{o}}(a * y)$ by (3). So

$$
\begin{gathered}
x x^{o}(a * y) \mathcal{R} x x^{o}(a * y)(a * y)^{o} x^{o o} x^{o}=e, \\
x^{\prime} x^{o}\left(a^{\prime} * y^{\prime}\right) \mathcal{R} x^{\prime} x^{o}\left(a^{\prime} * y^{\prime}\right) s x^{o o} x^{o}=f .
\end{gathered}
$$

Thus

$$
\begin{aligned}
e & =x x^{o}(a * y) y^{o} y^{\prime} s a^{\prime} a^{o} x^{o o} x^{o} \\
& =x x^{o}\left(a * y y^{o} y^{\prime}\right) s x^{o o} x^{o} \quad\left(a^{\prime} a^{o} x^{o o}=x^{o o} \text { since } a^{\prime} \in L\right) \\
& =x^{\prime} x^{o}\left(a * y^{\prime}\right) s x^{o o} x^{o} \quad\left(x x^{o}=x^{\prime} x^{o} \text { and } y^{\prime} \mathcal{R} y \mathcal{R} y y^{o}\right) \\
& =x^{\prime} x^{o}\left(a^{\prime} * y^{\prime}\right) s x^{o o} x^{o} \quad\left(x^{o} \in S^{o} \text { and } x^{o} a=x^{o} a^{\prime}\right) \\
& =f .
\end{aligned}
$$

Therefore $R_{x x^{o}(a * y)}=R_{x^{\prime} x^{o}\left(a^{\prime} * y^{\prime}\right)}$. Dually we have $L_{(a * y) y^{o} b}=L_{\left(a^{\prime} * y^{\prime}\right) y^{o} b^{\prime}}$. 
LEMMA 3.3. The set $\Gamma$ is a semigroup.

Proof. Let $e, f, g \in \Gamma$, where $e=\left(K_{x}, L_{a}\right), f=\left(K_{x_{1}}, L_{a_{1}}\right), g=\left(K_{x_{2}}, L_{a_{2}}\right)$. Then

$$
\begin{aligned}
(e f) g & =\left(K_{x x^{o}\left(a * x_{1}\right)}, L_{\left(a * x_{1}\right) x_{1}^{o} a_{1}}\right)\left(K_{x_{2}}, L_{a_{2}}\right) \\
& =\left(K_{x x^{o}\left(a * x_{1}\right)\left(a * x_{1}\right)^{o} x^{o o} x^{o}\left(\left(\left(a * x_{1}\right) x_{1}^{o} a_{1}\right) * x_{2}\right)}, L_{\left(\left(\left(a * x_{1}\right) x_{1}^{o} a_{1}\right) * x_{2}\right) x_{2}^{o} a_{2}}\right) \\
& =\left(K_{x x^{o}\left(a * x_{1}\right) x_{1}^{o}\left(a_{1} * x_{2}\right)}, L_{\left.\left(a * x_{1}\right) x_{1}^{o}\left(a_{1} * x_{2}\right) x_{2}^{o} a_{2}\right) .}\right.
\end{aligned}
$$

On the other hand,

$$
\begin{aligned}
e(f g) & =\left(K_{x}, L_{a}\right)\left(K_{x_{1} x_{1}^{o}\left(a_{1} * x_{2}\right)}, L_{\left(a_{1} * x_{2}\right) x_{2}^{o} a_{2}}\right) \\
& =\left(K_{x x^{o}\left(a * x_{1}\right) x_{1}^{o}\left(a_{1} * x_{2}\right)}, L_{\left(a * x_{1}\right) x_{1}^{o}\left(a_{1} * x_{2}\right) x_{2}^{o} a_{2}}\right) .
\end{aligned}
$$

Therefore $(e f) g=e(f g)$.

Lemma 3.4. Let $W=\left\{\left(K_{x}, L_{x}\right) \mid x \in S^{o}\right\}$. Then $W$ is an orthodox subsemigroup of $\Gamma$ isomorphic to $S^{o}$.

PROOF. We only need to notice that, for $x, y \in S^{o},\left(K_{x}, L_{x}\right)=\left(K_{y}, L_{y}\right)$ if and only if $x=y$.

Lemma 3.5. Let $e=\left(K_{x}, L_{a}\right)$. Put

$$
M(e)=\left\{\left(K_{x^{o}}, L_{x^{o}}\right) \in W \mid x^{o} \in V_{S^{o}}(x)\right\} .
$$

Then $V_{W}(e)=M(e)$.

Proof. Take $f=\left(K_{x^{o}}, L_{x^{o}}\right) \in W$, where $x^{o} \in V_{S^{o}}(x)$. Then

$$
\begin{aligned}
& \left(K_{x}, L_{a}\right)\left(K_{x^{o}}, L_{x^{o}}\right)\left(K_{x}, L_{a}\right)=\left(K_{x x^{o}\left(a * x_{o}\right) x^{o o}\left(x^{o} * x\right)}, L_{\left(a * x^{o}\right) x^{o o}\left(x^{o} * x\right) x^{o} a}\right)
\end{aligned}
$$

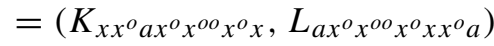

$$
\begin{aligned}
& =\left(K_{x}, L_{a}\right) \text {. }
\end{aligned}
$$

Also

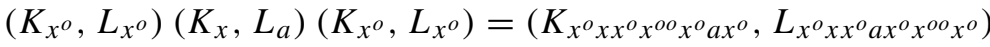

$$
\begin{aligned}
& =\left(K_{x^{o}}, L_{x^{o}}\right) \text {. }
\end{aligned}
$$

Thus $f \in V_{W}(e)$.

Conversely, let $f=\left(K_{y^{o}}, L_{y^{o}}\right) \in V_{W}(e)$, then $e f e=e, f e f=f$. So

$$
\begin{gathered}
\left(K_{x}, L_{a}\right)\left(K_{y^{o}}, L_{y^{o}}\right)\left(K_{x}, L_{a}\right)=\left(K_{x x^{o} a y^{o} x}, L_{a y^{o} x x^{o} a}\right)=\left(K_{x}, L_{a}\right), \\
\left(K_{y^{o}}, L_{y^{o}}\right)\left(K_{x}, L_{a}\right)\left(K_{y^{o}}, L_{y^{o}}\right)=\left(K_{y^{o} x x^{o} a y^{o}}, L_{y^{o} x x^{o} a y^{o}}\right)=\left(K_{y^{o}}, L_{y^{o}}\right) .
\end{gathered}
$$

Therefore $x=x x^{o} a y^{o} x$ since $x$ and $x x^{o} a y^{o} x$ have a common inverse by $T\left(x x^{o} a y^{o} x\right)=T(x)$. Similarly $y^{o}=y^{o} x x^{o} a y^{o}$. Then $x$ has an inverse

$$
x^{\#}=x^{o} y^{o o} x^{o} x^{o o} x^{o}=x^{o} y^{o o} x^{o} .
$$


On the other hand, $x^{o} y^{o o} x^{o} \in V_{S^{o}}\left(x y^{o} x\right)$; thus $x$ and $x y^{o} x$ have a common inverse and so $x=x y^{o} x$. Similarly $y^{o}=y^{o} x y^{o}$. Hence $y^{o} \in V_{S^{o}}(x)$ and therefore $f \in M(e)$. Now the proof of the lemma is completed.

\section{LEMMA 3.6. The set $W$ is a quasi-ideal orthodox transversal of $\Gamma$.}

PROOF. Take $e=\left(K_{x}, L_{a}\right) \in \Gamma$, and $x^{o} \in V_{S^{o}}(x) \cap V_{S^{o}}(a)$. It follows from Lemma 3.5 that $V_{W}(e) \neq \emptyset$, and hence condition (1.1) holds. To check condition (1.2), take $f=\left(K_{y}, L_{y}\right) \in W$, where $y \in S^{o}$. Then $e f=\left(K_{x x^{o} a y}, L_{a y}\right)$ since $x x^{o}(a * y)=$ $x x^{o}$ ay and $(a * y) y^{o} y=a y y^{o} y=a y$ by the assumption $y \in S^{o}$. Now let

$$
e^{\prime}=\left(K_{x^{o}}, L_{x^{o}}\right) \in V_{W}(e), \quad f^{\prime}=\left(K_{y^{o}}, L_{y^{o}}\right) \in V_{W}(f) .
$$

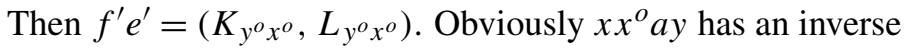

$$
\left(x x^{o} a y\right)^{\#}=y^{o} x^{o} x^{o o} x^{o}=y^{o} x^{o} .
$$

That is to say, $\left(K_{y^{o} x^{o}}, L_{y^{o} x^{o}}\right) \in M(e f)$ and thus $f^{\prime} e^{\prime} \in V_{W}(e f)$. Similarly we have $e^{\prime} f^{\prime} \in V_{W}(f e)$. Hence condition (1.2) holds and $W$ is an orthodox transversal of $\Gamma$.

Take $w_{1}, w_{2} \in W$ and $s \in \Gamma$. It is a routine matter to show that $w_{1} s w_{2} \in W$, so $W$ is a quasi-ideal of $\Gamma$.

Now we turn to prove the converse part of Theorem 3.1. Let $S$ be a regular semigroup and $S^{o}$ a quasi-ideal orthodox transversal of $S$. Let $R$ and $L$ be described as in Theorem 2.1. Then $R$ and $L$ are orthodox semigroups with an orthodox transversal $S^{o}$ which is a right ideal of $R$ and a left ideal of $L$. For every $(a, x) \in L \times R$, put $a * x=a x$. Then $a * x=a x=a^{o o} a^{o} a x x^{o} x^{o o} \in S^{o}$ since $S^{o}$ is a quasi-ideal of $S$. Clearly the map satisfies (1) and (2). By Theorem 2.5 the condition (3) holds. Therefore we get a regular semigroup $\Gamma$ in the same way as in the first part of Theorem 3.1. Finally we shall prove that $\Gamma$ is isomorphic to $S$.

Let $\left(K_{x}, L_{a}\right) \in \Gamma$. Define $\theta: \Gamma \longrightarrow S$ by $\left(K_{x}, L_{a}\right) \theta=x x^{o} a$, where $x^{o} \in V_{S^{o}}(x)$. It is evident that, for every $y^{o} \in V_{S^{o}}(x), x x^{o} a=x y^{o} a$ since $x x^{o} a \mathcal{H} x y^{o} a$ and

$$
y^{o} x x^{o} \in V\left(x x^{o} a\right) \cap V\left(x y^{o} a\right) .
$$

We first have to show that $\theta$ is well defined. If $\left(K_{x}, L_{a}\right)=\left(K_{y}, L_{b}\right)$ then $R_{x}=R_{y}$, $V_{S^{o}}(x)=V_{S^{o}}(y), L_{a}=L_{b}$ and so

$$
\begin{gathered}
x x^{o} a \mathcal{R} x x^{o} \mathcal{R} x \mathcal{R} y \mathcal{R} y y^{o} \mathcal{R} y y^{o} b, \\
x x^{o} a \mathcal{L} x^{o} a \mathcal{L} a \mathcal{L} b \mathcal{L} y^{o} b \mathcal{L} y y^{o} b .
\end{gathered}
$$

Thus $x x^{o} a \mathcal{H} y y^{o} b$ and we also have

$$
y^{o} x x^{o} \in V\left(x x^{o} a\right) \cap V\left(y y^{o} b\right) .
$$

Therefore $x x^{o} a=y y^{o} b$ since no $\mathcal{H}$-class contains more than one inverse of some element. 
Take $\left(K_{x}, L_{a}\right),\left(K_{y}, L_{b}\right) \in \Gamma$. Then

$$
\begin{aligned}
\left(\left(K_{x}, L_{a}\right)\left(K_{y}, L_{b}\right)\right) \theta & =\left(K_{x x^{o} a y}, L_{a y y^{o} b}\right) \theta \\
& =x x^{o} a y(a y)^{o} a^{o o} a^{o}(a y) y^{o} b \\
& =x x^{o} a y y^{o} b \\
& =\left(K_{x}, L_{a}\right) \theta\left(K_{y}, L_{b}\right) \theta,
\end{aligned}
$$

and $\operatorname{so} \theta$ is a homomorphism.

For every $x \in S$,

$$
\begin{gathered}
x x^{o} x^{o o} \in R, \quad x^{o o} x^{o} x \in L \quad \text { and } \quad x^{o} \in V_{S^{o}}\left(x x^{o} x^{o o}\right) \cap V_{S^{o}}\left(x^{o o} x^{o} x\right), \\
\left(K_{x x^{o} x^{o o}}, L_{x^{o o} x^{o} x}\right) \theta=x x^{o} x^{o o} \cdot x^{o} \cdot x^{o o} x^{o} x=x .
\end{gathered}
$$

Therefore $\theta$ is surjective.

Now let $\left(K_{x}, L_{a}\right),\left(K_{y}, L_{b}\right) \in \Gamma$ such that $\left(K_{x}, L_{a}\right) \theta=\left(K_{y}, L_{b}\right) \theta$, that is $x x^{o} a=$ $y y^{o} b$. So

$$
x \mathcal{R} x x^{o} \mathcal{R} x x^{o} a=y y^{o} b \mathcal{R} y y^{o} \mathcal{R} y
$$

and

$$
a \mathcal{L} x^{o} a \mathcal{L} x x^{o} a=y y^{o} b \mathcal{L} y^{o} b \mathcal{L} b .
$$

That is $R_{x}=R_{y}$ and $L_{a}=L_{b}$. It is easy to see that $x^{o} \in V_{S^{o}}\left(x x^{o} a\right)$ and $y^{o} \in$ $V_{S^{o}}\left(y y^{o} b\right)$, so

$$
V_{S^{o}}(x)=V_{S^{o}}\left(x x^{o} a\right)=V_{S^{o}}\left(y y^{o} b\right)=V_{S^{o}}(y) .
$$

Hence $\theta$ is injective.

\section{Acknowledgements}

The first author expresses his sincere thanks to Professor M. Jackson and the referees for their important and constructive modifying suggestions.

\section{References}

[1] T. S. Blyth and M. H. Almeida Santos, 'A classification of inverse transversal', Comm. Algebra 29(2) (2001), 611-624.

[2] _ 'Amenable orders associated with inverse transversals', J. Algebra 240(1) (2001), 143-164.

[3] T. S. Blyth and R. B. McFadden, 'Regular semigroups with a multiplicative inverse transversal', Proc. Roy. Soc. Edinburgh A 92 (1982), 253-270.

[4] J. Chen, 'On regular semigroups with orthodox transversals', Comm. Algebra 27 (1999), $4275-4288$.

[5] J. Chen and Y. Guo, 'Orthodox transversals of regular semigroups', Internat. J. Algebra Comput. 11(2) (2001), 269-279.

[6] J. M. Howie, An Introduction to Semigroup Theory (Academic Press, London, 1976).

[7] X. Kong, 'Regular semigroups with quasi-ideal orthodox transversals', Semigroup Forum $\mathbf{7 4}$ (2007), 247-258. 
[8] D. B. McAlister and R. B. McFadden, 'Regular semigroups with inverse transversals', Q. J. Math. 34(2) (1983), 459-474.

[9] T. Saito, 'Structure of regular semigroups with a quasi-ideal inverse transversal', Semigroup Forum 31 (1985), 305-309.

[10] _ 'A note on regular semigroups with inverse transversals', Semigroup Forum 33 (1986), $149-152$.

[11] X. Tang, 'Regular semigroups with inverse transversals', Semigroup Forum 55 (1997), 25-32.

XIANGJUN KONG, School of Mathematical Sciences, Qufu Normal University, Qufu, Shandong 273165, People's Republic of China

and

Current address: Department of Mathematics, Lanzhou University, Lanzhou,

Gansu 730000, People's Republic of China

e-mail: xiangjunkong97@163.com

XIANZHONG ZHAO, Department of Mathematics, Northwest University, Xi'an, Shaanxi 710069, People's Republic of China 